\title{
EXPERIENCIAS EXITOSAS EN REDES TEMÁTICAS DE DOCENCIA IBEROAMERICANAS EN LA FACULTAD DE CIENCIAS ADMINISTRATIVAS
}

\author{
Ebor Fairlie Frisancho (*) \\ E-mail:efairlie@gmail.com
}

\begin{abstract}
RESUMEN
El presente artículo pretende dar a conocer a la comunidad académica de la Facultad de Ciencias Administrativas la importancia de pertenecer y conformar Redes Tematicas de Docencia con la culminación exitosa de dos redes temáticas de Turismo y Desarrollo y Medio Ambiente, conformadas en el año 2007 entre la Corporación Universitaria del Meta (Colombia), la Universidad de Panamá (Panamá) y la Universidad Nacional Mayor de San Marcos (Perú) con la realización de cuatro Congresos Internacionales con docentes ponentes de dichas universidades que viajaron subvencionados íntegramente con las ayudas que provee el Grupo de Universidades Iberoamericanas La Rábida, auspiciadas por la Universidad Internacional de Andalucía (España), y la emisión de Memorias de los congresos que incluyen la publicación de cada ponencia.
\end{abstract}

Palabras claves: Redes Temáticas, Docencia, Turismo, Desarrollo y Medio Ambiente, Corporación Universitaria del Meta, Universidad de Panama, Grupo La Rábida.

\begin{abstract}
This article aims to inform the academic community of Administrative Sciences Faculty, the importance of belonging and shape Subject Teaching Networks with the successful completion of two thematic networks; Tourism and Development and the Environment, formed in 2007 between the Corporación Universitaria del Meta (Colombia), Universidad de Panamá (Panamá) and the Universidad Nacional Mayor de San Marcos (Perú) with the implementation of four International Conferences with professors from these universities who traveled fully supported with aid provided by the Group of Latin American Universities of La Rabida, sponsored by Universidad Internacional de Andalucía (España) and the issue of Proceedings of the conference, including the publication of each paper.
\end{abstract}

Key words: Thematic Networks, Teaching, Tourism, Environment and Development, Corporación Universitaria del Meta, Universidad de Panama, Grupo La Rábida.

(*) Magister en Administración, Licenciado en Administración de Empresas UNMSM. Profesor Investigador del Instituto de Investigaciones. Asesor de la Oficina de Calidad Académica y Acreditación. Coordinador Académico de la Unidad de Postgrado de la Facultad de Ciencias Administrativas. Docente de postgrado en diversas universidades del interior del país. Coordinador de Redes Temáticas de Docencia Iberoamericanas. 


\section{INTRODUCCIÓN}

Desde que en el año 2007 la Universidad Nacional Mayor de San Marcos, a través de la Facultad de Ciencias Administrativas, se presentó con dos proyectos de Redes Temáticas de Docencia junto con la Corporación Universitaria del Meta (Colombia) y la Universidad de Panamá (Panamá) a la convocatoria de Ayudas a Redes Temáticas de Docencia auspiciadas por el Grupo de Universidades Iberoamericanas La Rábida y resultaran seleccionados dichos proyectos, nuestra Facultad se ha visto comprometida como integrante de las redes temáticas de docencia GALEON (Turismo) y ECOBIOS (Desarrollo y Medio Ambiente) de La Rábida, promovidas por la Universidad Internacional de Andalucía, España.

Es de esta forma que nuestra Facultad se ha visto favorecida con cuatro viajes a los hermanos países de Colombia y Panamá para realizar ponencias sobre los temas de Turismo y Desarrollo y Medio Ambiente durante los años 2008 y 2009, con todos los gastos pagados, beneficiando a aquellos colegas que viajaron para exponer sus investigaciones versadas en dichos temas

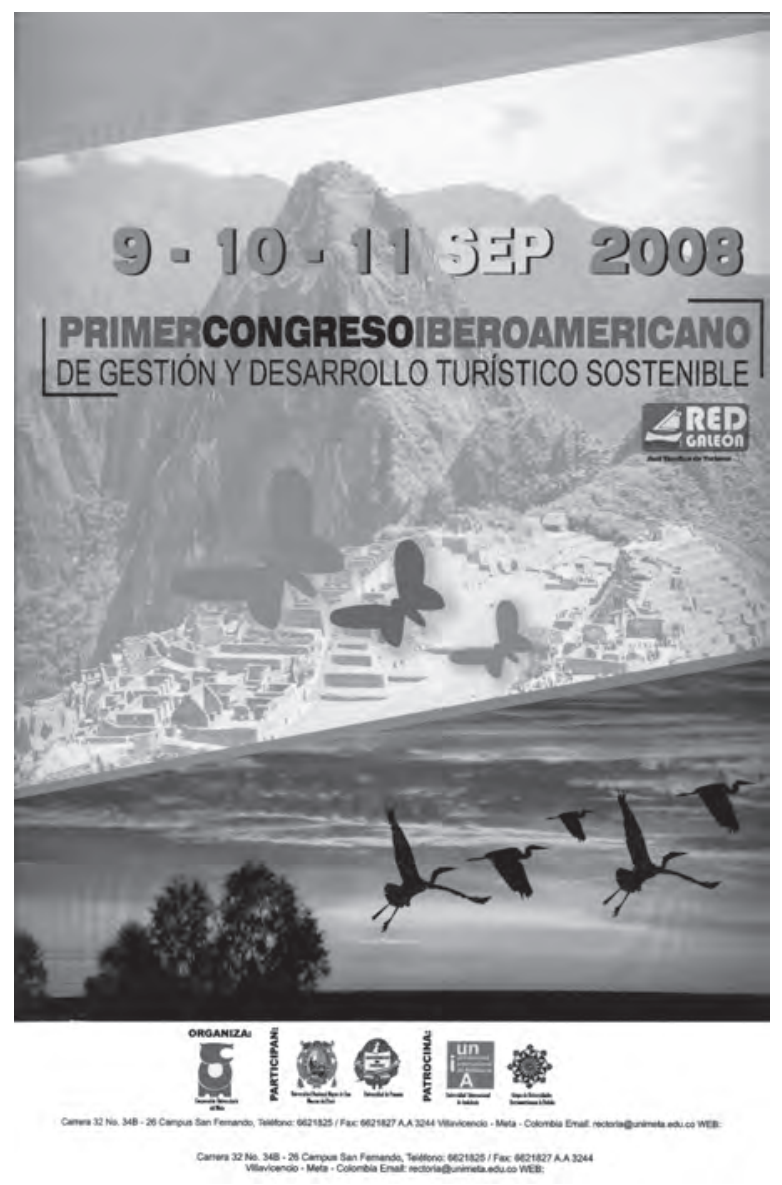

\section{REDES TEMÁTICAS}

De acuerdo al Programa Iberoamericano de Ciencia y Tecnología para el Desarrollo (CYTED), define a la red temática como "Asociaciones de grupos de investigación de entidades públicas o privadas de los países miembros del Programa CYTED, cuyas actividades científicas o tecnológicas están relacionadas dentro de un ámbito común de interés". De acuerdo a este mismo programa, se pretende que estos grupos tengan:

- Interacciones científicas estables y continuadas.

- Intercambios de conocimiento científico y técnico de mutuo interés.

- Potenciación sinérgica y coordinación de sus líneas de I+D.

- Intercambios y movilidad del personal de investigación.

- Formación de recursos humanos.

- Capacitación técnica y metodológica.

- Elaboración de propuestas de posibles Proyectos de Investigación o Proyectos de Innovación IBEROEKA.

- Acciones de difusión y transferencia tecnológica entre grupos o entidades distintas, siempre que sean técnica, económica y comercialmente viables.

Tienen como objetivo principal el intercambio de conocimientos entre grupos de investigación y la potenciación de la cooperación como método de trabajo. Su misión no es obtener un resultado tangible, ya sea producto, proceso o servicio, sino crear un marco de colaboración en el trabajo que permita que en el futuro puedan surgir nuevas acciones conjuntas: proyectos de investigación, proyectos de innovación IBEROEKA u otras actividades relacionadas con la $\mathrm{I}+\mathrm{D}+\mathrm{I}$.

El mantenimiento y consolidación de las redes temáticas requiere la participación activa de todos los grupos integrantes, la percepción de la existencia de un beneficio mutuo y de una mejora de la competencia de cada uno de ellos. Por esta razón, la identificación de los temas, la selección de los grupos y la idoneidad de las actividades programadas son elementos clave para su éxito y sostenibilidad.

Como principales características de las redes temáticas destacan las siguientes: 
- Publicar y difundir, antes de su finalización, al menos una monografía sobre el "estado del arte" del tema o temas tratados.

- Estar conformadas por un mínimo de seis participantes de al menos seis países miembros del Programa, valorándose positivamente la mayor cobertura geográfica posible.

- Duración máxima de cuatro años.

Como ejemplos de redes temáticas para el CYTED se pueden citar las siguientes:

- Red Iberoamericana sobre la conservación de la biodiversidad de los animales domésticos locales para el desarrollo rural sostenible.

- Red Iberoamericana para el desarrollo de tecnologías poscosecha y de gestión para la comercialización de frutas y verduras de producción integrada.

- Red Iberoamericana de genética molecular aplicada a la medicina forense.

- Red Iberoamericana sobre mostradores de cristal líquido.

Para el caso de este Programa, se indica que en las redes temáticas pueden participar:

- Centros públicos de I+D: Universidades públicas, organismos públicos de investigación reconocidos como tales por la Ley, y cualquier otro centro de I $+\mathrm{D}$ dependiente de las administraciones públicas.

- Centros privados de I+D sin ánimo de lucro: Universidades y entidades privadas sin ánimo de lucro, con capacidad y actividad demostrada en acciones de I+D. Quedan incluidos los centros tecnológicos cuya propiedad y gestión sea mayoritariamente de las administraciones públicas.

- Centros tecnológicos: Centros de innovación y tecnología cuya propiedad u órgano de gobierno no sea mayoritaria de las administraciones públicas.

- Sector privado: Empresas interesadas en mantener un marco de cooperación en materia de ciencia y tecnología para el desarrollo.

El proponente o coordinador debe ser una persona física perteneciente a alguna institución de uno de los países signatarios al Programa CYTED.
Los grupos de investigación que participen en la propuesta deberán estar liderados por un investigador principal.

Otra propuesta de redes temáticas la tiene la Red Colombiana de Semilleros de Investigación, en donde indica que son asociaciones de Unidades de Investigación y son la integración de semilleros en cada uno de los nodos alrededor de diferentes temas acerca de investigación formativa, ciencia o tecnología y emprendimiento empresarial, cuyas actividades están relacionadas con un tema específico seleccionado para la red.

Tienen como objetivo el intercambio de conocimientos existentes entre semilleros de investigación y la potenciación de la cooperación como método de trabajo.

De acuerdo a esta red, las redes temáticas pretenden propiciar entre los semilleros las siguientes acciones:

- Interacciones investigativas estables y continuadas.

- Intercambios de conocimiento científico y técnico de mutuo interés.

- Potenciación sinérgica y coordinación de sus líneas de I+D.

- Intercambios y movilidad del personal de investigación.

- Formación de recursos humanos.

- Capacitación técnica y metodológica.

- Elaboración de propuestas al Gestor de Área de posibles proyectos de investigación o de innovación.

- Acciones de difusión y transferencia tecnológica entre semilleros o redes distintas, siempre que sean técnica, económica y comercialmente viables.

Los resultados esperados al finalizar la red son la participación activa de todas las Unidades Investigativas Asociadas (semilleros, redes institucionales, redes locales, nodos) y la percepción de la existencia de un beneficio mutuo y de una mejora de la competencia de cada una de estas unidades.

Su misión no es necesariamente un resultado tangible, ya sea producto, proceso o servicio, sino crear un marco colaborativo de trabajo donde todos ganen, y donde en el futuro puedan surgir 
nuevas acciones conjuntas, como Proyectos de Investigación, Proyectos de Innovación u otras actividades externas relacionadas con la $\mathrm{I}+\mathrm{D}+\mathrm{I}$ o generación de posibles Unidades de Emprendimiento Empresarial.

Por ello, la identificación de los temas y la idoneidad de las actividades programadas son elementos claves para el éxito y persistencia de las redes temáticas. Se espera que al final de cada semestre presenten un artículo que dé cuenta de los documentos tratados y de las reflexiones o conceptualizaciones a las que se ha llegado, luego de un trabajo comprometido y de encuentros presenciales y/o virtuales por los menos una vez a la semana.

Antes del segundo año de ejecución deberán publicar al menos una monografía sobre el "estado del arte" del tema o temas tratados por la red. Las Unidades Investigativas Asociadas son los elementos dinámicos de las redes temáticas. Cada unidad está constituida por integrantes de diferentes semilleros de investigación de una misma institución, red local o nodo, que desarrollan actividades de I+D en el tema de la red y se comprometen a participar activamente en las diversas actividades programadas.

Cada red debe tener un mínimo de tres Unidades Investigativas Asociadas (Semilleros) de diferentes instituciones y la mayor cobertura posible en el nodo. Las redes pueden adoptar a nivel interno la organización que consideren apropiada en función de los objetivos perseguidos y de las actividades a desarrollar. Las redes temáticas tendrán una duración de dos años y la prórroga anual estará condicionada a la dinámica demostrada y al grado de cumplimiento de sus objetivos. El Comité Académico de cada nodo puede aprobar el carácter permanente de una red, cuando ello sea aconsejable por su contenido y número de semilleros participantes.

Otra definición interesante lo demuestra la Red Vegetalia (G03/094) adscrita al Instituto de Salud Carlos III en donde indica que una red temática interdisciplinar está constituida por un conjunto de nodos donde se integran investigadores y recursos que, con una ubicación física concreta y adscritos a un Centro de Investigación, se comprometen a desarrollar un tema, generar conocimiento de forma planificada o a trabajar en la solución de un problema de $\mathrm{I}+\mathrm{D}$ de carácter científico y/o tecnológico amplio pero bien definido, mediante la interacción de sus respectivas contribuciones y explotando al máximo las sinergias derivadas de las mismas.

Existen tres circunstancias que pueden aconsejar la creación de una red temática:

- La existencia de un problema científico donde la mejor forma de abordarlo es una aproximación interdisciplinar. La experiencia y conocimientos de la mayor parte de las disciplinas necesarias pueden existir, pero se trata de estimular su comunicación y conexión para concentrarlas en torno al problema concreto.

- Cuando la experiencia y conocimientos para abordar un problema concreto aparece disgregada en varios centros, sin conexión ninguna, incluso aunque la materia no sea interdisciplinar. En este caso se trataría de nuclear esos esfuerzos, de forma que la red actúe de centro virtual.

- La existencia de un problema concreto o una línea temática para la que no existe capacidad o masa crítica suficiente pero que una vez realizados análisis de pertinencia, oportunidad y viabilidad, se considera conveniente alcanzar esa masa crítica estructurando una red temática para disponer de la necesaria flexibilidad de planteamiento y movilidad de recursos que previsiblemente el tema requiere.

Los factores que han favorecido la creación y desarrollo de las redes temáticas en el ámbito sanitario español han sido:

- El Plan Nacional de Investigación Científica, Desarrollo e Innovación Tecnológica $(\mathrm{I}+\mathrm{D}+\mathrm{I})$ para el período 2000-2003. En el área de Biomedicina prevé diversas modalidades de participación de los distintos organismos públicos con funciones de investigación, entre otros la creación de redes de investigación Biomédica.

- La existencia de un clima alentador en las directrices emanadas del Ministerio de Sanidad y Consumo.

- La necesidad de adaptar la política científica en biomedicina al Programa Marco de Investigación y Desarrollo Tecnológico de la Unión Europea (2002-2006), en el que se contemplan y potencian las denominadas redes científicas de excelencia.

- La creación de un fondo para la investigación con la finalidad de financiar proyectos de 
investigación de interés general basados en el acuerdo suscrito entre el ministerio de Sanidad y Consumo y Farmaindustria para la elaboración y ejecución de un plan integral de medidas de control del gasto farmacéutico y uso racional del medicamento.

De acuerdo a esta red, los requisitos necesarios para la constitución de una red temática de investigación cooperativa se basan en la orden SCO/709/2002, del 22 de marzo ("Boletín Oficial del Estado del 3 de abril") que establece las normas que regulan su funcionamiento así como la convocatoria mediante concurso en régimen de publicidad, objetividad y concurrencia competitiva. En dicha orden se expresan los siguientes requisitos:

- Compromiso de asociación de los nodos a través de la conformidad de sus representantes legales en la solicitud.

- Las entidades que pueden componer estas redes serán necesariamente centros públicos y/o privados de I + D + I. A estos efectos se entiende por privados de $I+D+I$ a las entidades e instituciones sanitarias privadas, universidades y otras entidades privadas, con

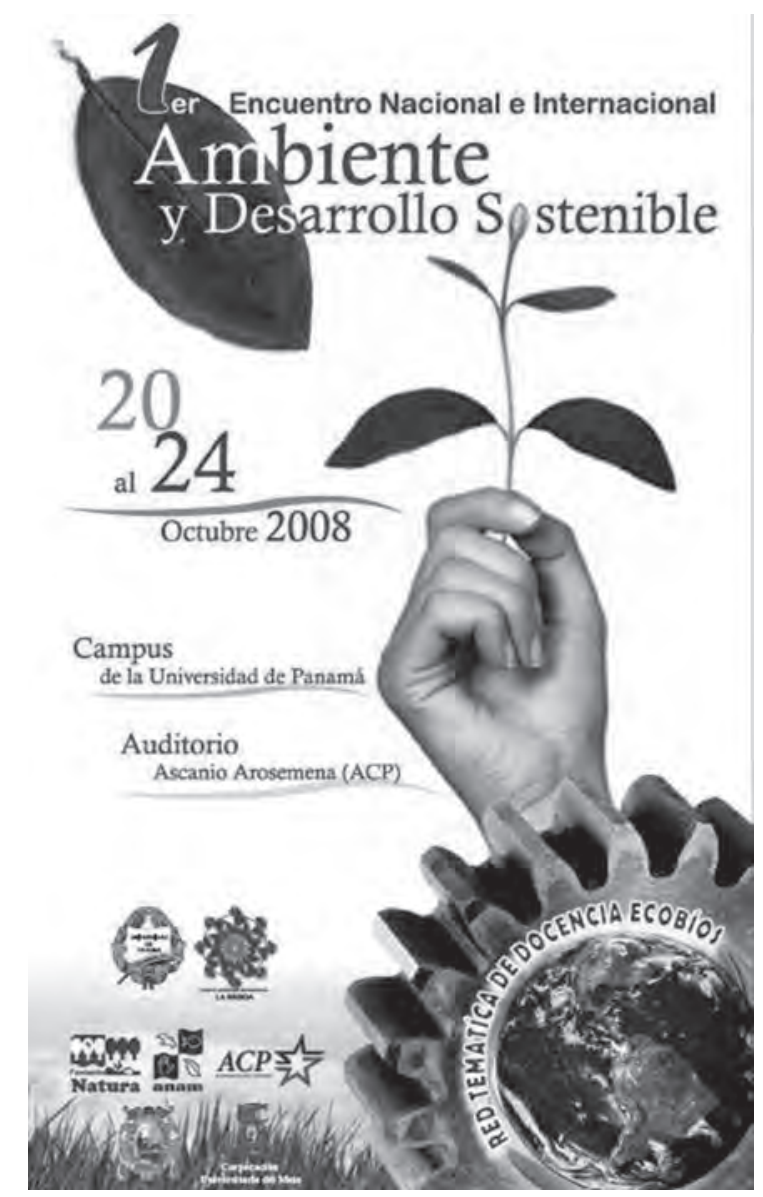

personalidad jurídica propia y sin ánimo de lucro, con capacidad o actividad demostradas en acciones de $\mathrm{I}+\mathrm{D}+\mathrm{I}$. En caso de tener ánimo de lucro, deberán estar incluidos en acuerdos específicos con las administraciones sanitarias en el ámbito de los objetivos recogidos en la presente convocatoria.

- Existencia de una estructura organizativa determinada donde estén representados todos los centros o unidades conformantes de la red.

- Diseño de un Plan Estratégico conjunto de investigación cooperativa de interés general donde se evidencie un proyecto científico conjunto de tres años de duración, con la participación de los diferentes centros o unidades donde se verifique la interacción intelectual en investigación, formación o práctica clínica, señalando las áreas, objetivos y líneas científicas comunes así como las distintas actuaciones y acciones necesarias para conseguir los objetivos propuestos. Dicho plan hará hincapié en la integración de los distintos tipos de investigación existentes. En todas las redes deben participar al menos un centro o unidad de investigación emergente que se constituya como nodo. El proyecto científico presentado podrá ser financiado total o parcialmente. Igualmente se incluirán los gastos necesarios para el funcionamiento de la red.

- Configuración de un Comité Científico Externo que evalúe la calidad de la actividad científica cooperativa o colaborativa. Este comité no es necesario en las redes de grupos.

- Disponer de bases de datos y registros compartidos así como, en su caso, de bancos de tejidos colaborativos.

- Diseño y desarrollo de un Plan de Formación en Investigación vinculado, preferentemente, a programas de tercer ciclo.

- Disponer de una guía escrita que asegure la calidad, la ética y la buena práctica científica en investigación, y el cumplimiento de las disposiciones legales y los principios comúnmente aceptados en este ámbito de actuación.

- Disponer de un registro con la filiación del personal que participa en las actividades de la red, proveniente de los distintos centros o unidades de investigación y su vinculación a los mismos. 
- Disponer de un registro de los datos y las actividades de I+D+I desarrolladas al amparo de la red, que esté protegido conforme a la legislación vigente en esta materia.

- Por cada nodo o centro existirá un coordinador científico responsable a efectos científicos. El coordinador científico deberá poseer el título de Doctor, y deberá acreditar experiencia en actividades de I+D+I durante al menos diez años o, alternativamente, que haya dirigido proyectos de investigación durante al menos cinco años y pertenecer a la plantilla del centro a través del cual se solicita la ayuda.

- En las redes figurará como coordinador científico de la misma uno de los coordinadores científicos de los centros conformantes de la red. El coordinador científico de la red será el responsable a todos los efectos de la coordinación científica y seguimiento de la red.

\section{REDES TEMÁTICAS DE DOCENCIA}

Para nuestro caso, la Universidad Nacional Mayor de San Marcos se encuentra suscrita al Grupo de Universidades Iberoamericanas La Rábida, organismo sin fines de lucro, fundado por las Universidades Iberoamericanas firmantes del Convenio de Cooperación firmado en el año 1995 en la Sede Iberoamericana Santa María de la Rábida de la Universidad Internacional de Andalucía y cuya finalidad es la cooperación académica, científica, tecnológica y cultural, para estrechar los lazos universitarios y la integración de los pueblos iberoamericanos.

Para el logro de sus fines, las actividades del Grupo de Universidades La Rábida estarán dirigidas al cumplimiento de los siguientes objetivos:

- Establecer y estrechar vínculos de colaboración en la búsqueda de un espacio común académico y estudiantil, que posibilite el desarrollo progresivo y sostenido de las universidades que lo integran.
- Constituirse en un instrumento que propicie, con el esfuerzo mancomunado de sus miembros, la realización de actividades que favorezcan a sus respectivos pueblos en la esfera de la cultura, la educación, la ciencia y la técnica.

- Promover y formular propuestas de colaboración a partir de las relaciones bilaterales y multilaterales desarrolladas por sus miembros.

- Coordinar acciones que contribuyan al cumplimiento de los objetivos acordados.

- Promover el fortalecimiento institucional de las universidades en aspectos de mejoramiento curricular, gestión económica y administrativa.

- Promover y facilitar el intercambio de académicos e investigadores entre las universidades miembros del Grupo de Universidades La Rábida.

- Organizar cursos y postgrados regionales con reconocimiento pleno de sus miembros.

- Crear redes regionales de investigación y desarrollo con el objeto de elaborar proyectos conjuntos, compartir equipos y laboratorios.

- Desarrollar el intercambio de información y de experiencias que permitan multiplicar las posibilidades de cooperación entre sus miembros.

La pertenencia al grupo confiere el derecho a proponer a la UNIA la organización de maestrías y cursos de formación para celebrar tanto en España como en Iberoamérica; la conformación de redes temáticas de docencias entre ellas, además de ser destinatarias de los premios de investigación convocados por la UNIA y de las actividades aprobadas en la Asamblea General. Finalmente, sus estudiantes tienen preferencia para disfrutar de becas completas (alojamiento incluido) que la UNIA concede en cada uno de sus postgrados.

Este grupo lo componen, a la fecha, 61 universidades tanto públicas como privadas y son las siguientes:

\begin{tabular}{|l|l|}
\hline NOMBRE DE LA UNIVERSIDAD & PAÍS DE ORIGEN \\
\hline UNIVERSIDAD NACIONAL DE SALTA & ARGENTINA \\
\hline UNIVERSIDAD CATÓLICA DE SALTA & ARGENTINA \\
\hline UNIVERSIDAD NACIONAL DE CATAMARCA & ARGENTINA \\
\hline UNIVERSIDAD NACIONAL DE LA RIOJA & ARGENTINA \\
\hline UNIVERSIDAD NACIONAL DE LA PAMPA & ARGENTINA \\
\hline UNIVERSIDAD NACIONAL DE TUCUMÁN & ARGENTINA \\
\hline
\end{tabular}




\begin{tabular}{|c|c|}
\hline UNIVERSIDAD NACIONAL DE LA MATANZA & ARGENTINA \\
\hline UNIVERSIDAD NACIONAL DE RÍO CUARTO & ARGENTINA \\
\hline UNIVERSIDAD NACIONAL DE SANTIAGO DEL ESTERO & ARGENTINA \\
\hline UNIVERSIDAD AUTÓNOMA TOMÁS FRÍAS & BOLIVIA \\
\hline UNIVERSIDAD NUESTRA SEÑORA DE LA PAZ & BOLIVIA \\
\hline UNIVERSIDAD AUSTRAL DE CHILE & CHILE \\
\hline UNIVERSIDAD DEL MAR & CHILE \\
\hline UNIVERSIDAD DE SANTIAGO DE CHILE & CHILE \\
\hline UNIVERSIDAD DE ARTE Y CIENCIAS SOCIALES (ARCIS) & CHILE \\
\hline UNIVERSIDAD DEL BÍO-BÍO & CHILE \\
\hline UNIVERSIDAD CIENCIAS DE LA INFORMÁTICA & CHILE \\
\hline CORPORACIÓN UNIVERSITARIA DEL META & COLOMBIA \\
\hline ESCUELA DE INGENIERÍA DE ANTIOQUÍA & COLOMBIA \\
\hline ESUMER & COLOMBIA \\
\hline UNIVERSIDAD DE ANTIOQUÍA & COLOMBIA \\
\hline UNIVERSIDAD DE CARTAGENA & COLOMBIA \\
\hline UNIVERSIDAD NACIONAL ABIERTA Y A DISTANCIA & COLOMBIA \\
\hline UNIVERSIDAD SANTIAGO DE CALI & COLOMBIA \\
\hline UNIVERSIDAD DEL PACÍFICO & COLOMBIA \\
\hline UNIVERSIDAD MILITAR DE NUEVA GRANADA & COLOMBIA \\
\hline UNIVERSIDAD DE COSTA RICA & COSTA RICA \\
\hline INSTITUTO SUPERIOR POLITÉCNICO JOSÉ ANTONIO ECHEVERRÍA & CUBA \\
\hline UNIVERSIDAD CENTRAL DE LAS VILLAS & CUBA \\
\hline UNIVERSIDAD DE PINAR DEL RÍO & CUBA \\
\hline UNIVERSIDAD DE LA HABANA & CUBA \\
\hline UNIVERSIDAD TÉCNICA DEL NORTE & ECUADOR \\
\hline UNIVERSIDAD CATÓLICA DE SANTIAGO DE GUAYAQUIL & ECUADOR \\
\hline UNIVERSIDAD DE CUENCA & ECUADOR \\
\hline UNIVERSIDAD LAICA ELOY ALFARO DE MANABÍ & ECUADOR \\
\hline UNIVERSIDAD TÉCNICA ESTATAL DE QUEVEDO & ECUADOR \\
\hline UNIVERSIDAD TÉCNICA PARTICULAR DE LOJA & ECUADOR \\
\hline UNIVERSIDAD TECNOLÓGICA EQUINOCCIAL & ECUADOR \\
\hline UNIVERSIDAD ANDINA SIMÓN BOLÍVAR - SEDE ECUADOR & ECUADOR \\
\hline UNIVERSIDAD DON BOSCO & EL SALVADOR \\
\hline UNIVERSIDAD FRANCISCO GAVIDIA & EL SALVADOR \\
\hline UNIVERSIDAD DE SAN CARLOS DE GUATEMALA & GUATEMALA \\
\hline BENEMÉRITA UNIVERSIDAD AUTÓNOMA DE PUEBLA & MÉXICO \\
\hline UNIVERSIDAD DE GUADALAJARA & MÉXICO \\
\hline UNIVERSIDAD MICHOACANA SAN NICOLÁS DE HIDALGO & MÉXICO \\
\hline UNIVERSIDAD NACIONAL AUTÓNOMA DE NICARAGUA / LEÓN & NICARAGUA \\
\hline UNIVERSIDAD NACIONAL AGRARIA (NICARAGUA) & NICARAGUA \\
\hline UNIVERSIDAD DE PANAMÁ & PANAMÁ \\
\hline UNIVERSIDAD AUTONÓMA DE ASUNCIÓN & PARAGUAY \\
\hline PONTIFICIA UNIVERSIDAD CATÓLICA DEL PERÚ & PERÚ \\
\hline UNIVERSIDAD CATÓLICA DE SANTA MARÍA & PERÚ \\
\hline UNIVERSIDAD DE LIMA & PERÚ \\
\hline UNIVERSIDAD NACIONAL DE TUMBES & PERÚ \\
\hline UNIVERSIDAD NACIONAL MAYOR DE SAN MARCOS & PERÚ \\
\hline
\end{tabular}




\begin{tabular}{|l|l|}
\hline UNIVERSIDAD NACIONAL SAN ANTONIO ABAD DEL CUSCO & PERÚ \\
\hline UNIVERSIDAD DEL ZULIA & VENEZUELA \\
\hline UNIVERSIDAD CENTRAL DE VENEZUELA & VENEZUELA \\
\hline UNIVERSIDAD DE HUELVA (ASOCIADA) & ESPAÑA \\
\hline UNIVERSIDAD DE CÁDIZ (ASOCIADA) & ESPAÑA \\
\hline UNIVERSIDAD INTERNACIONAL DE ANDALUCÍA & ESPAÑA \\
\hline UNIVERSIDADE DO ALGARVE & PORTUGAL \\
\hline
\end{tabular}

Las temáticas de conformación de las redes, en el caso del año 2007, de acuerdo al Comité Ejecutivo del Grupo de Universidades Iberoamericanas La Rábida, fueron:

- Educación.

- Desarrollo y Medio Ambiente.

- Fortalecimiento Institucional.

- Turismo.

- Patrimonio y Cultura.

- Nuevas Tecnologías.

- Interculturalidad.

- Migraciones.

- Sociedad, Democracia y Estado.

- Acreditación Universitaria.

Lo que se encuentra resaltado son las temáticas en las que nuestra Facultad se presentó junto a otras dos universidades integrantes del Grupo.

Por lo general, estas redes convocan cada dos años, seis ayudas para la conformación de Redes Académicas de Docencia integradas por tres universidades del grupo que no sean del mismo país y que se articule una movilidad docente entre ellas, solventada íntegramente por la UNIA.

En el caso particular del Grupo La Rábida, la Universidad Internacional de Andalucía (UNIA) había previsto los fondos necesarios de hasta $€$ 5,000 anuales de cada ayuda para los años 2008 y 2009 que cubrirían los gastos de movilidad de los participantes, incluyendo pasajes aéreos y gastos de transporte que se generen hasta culminar el evento.

Los gastos de alojamiento, manutención y seguro de asistencia sanitaria de los docentes visitantes estarían a cargo de la universidad destino por los días que duró el evento.

Este evento debería incluir una actividad (seminario, simposium, congreso, etc.) como mínimo de 60 horas cada año, que sería realizado en al menos dos de las universidades del grupo participante. En nuestro caso, la Universidad Nacional Mayor de San Marcos fue visitante en Colombia y Panamá por la Red Galeón y por la Red Ecobios.

Los formatos para presentarse a estos proyectos se encuentran en la página web del Grupo La Rábida.

\section{RED GALEÓN}

La Red Temática GALEÓN de Gestión y Desarrollo Turístico Sostenible, se conforma de acuerdo a las bases establecidas en la Resolución de 11 de julio del 2007 del Presidente del Grupo de Universidades Iberoamericanas La Rábida por la que se convocan ayudas para redes temáticas

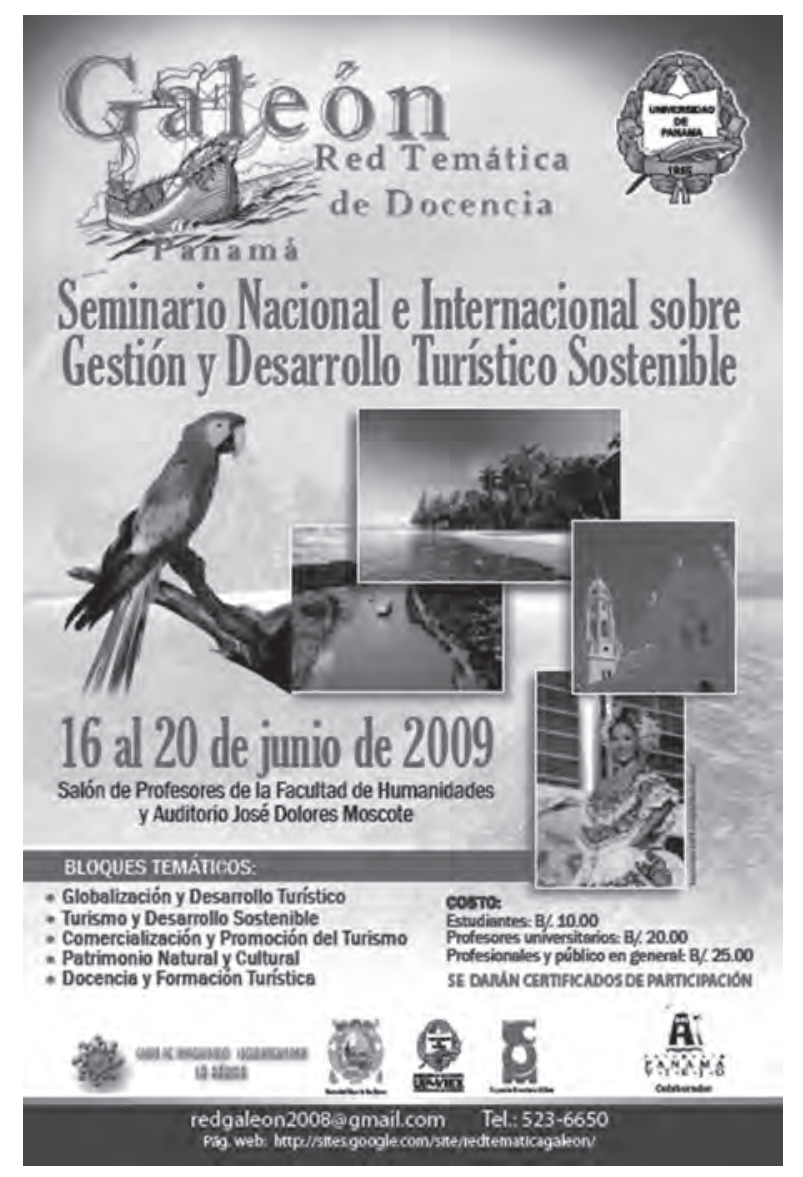


de docencia, a las cuales debe responder en sus características, objetivos, configuración y fines.

\section{Composición (universidades)}

- Corporación Universitaria del Meta (UNIMETA), Colombia (Coordinadora); sede donde se realizó la primera actividad docente

- Universidad de Panamá, Panamá; sede donde se realizó la segunda actividad docente

- Universidad Nacional Mayor de San Marcos, Perú.

La Red Temática de Docencia GALEÓN organizó un Congreso Iberoamericano sobre Gestión y Desarrollo Turístico Sostenible, durante el mes de agosto del 2008, en la Corporación Universitaria del Meta (UNIMETA), Colombia, y un Simposio en la Universidad de Panamá en el mes de septiembre del 2009 con el propósito de abrir espacios para la actualización y capacitación de los estudiantes y profesionales, y a los interesados por temáticas de turismo o que están desarrollando actividades propias del sector.

Con esta pretensión es que la red realizó estas actividades en donde especialistas en la materia presentaron un amplio debate alrededor de dos núcleos centrales como son la Gestión y el Desarrollo Turístico Sostenible, los cuales se desglosaron en otras líneas temáticas.

Las actividades se completaron con mesas de trabajo donde se consolidaron líneas de actuación, gestión y modelos de turismo sostenible, macroruedas de experiencias exitosas y la muestra de stands turísticos.

Para la realización de estos Congresos que la red ofreció se contó con el invaluable apoyo del Grupo de Universidades Iberoamericanas de La Rábida, en las ayudas que otorgan a las Redes Temáticas de Docencia.

\section{Objetivo general}

Analizar y afianzar los criterios según los cuales la gestión y el desarrollo turístico sostenible deben ser indisolublemente ligados para la satisfacción de las necesidades socioeconómicas actuales y futuras.

\section{Objetivos específicos}

- Conformar un grupo de profesores e investigadores iberoamericanos interesados en las líneas de Gestión y Desarrollo Turístico Sostenible para hacer rentables y duraderos estos intercambios académicos de países similares en su geografía, economía y grado de desarrollo. Estas actividades y reflexiones habrán de enriquecer a unos y a otros y sentar la base de futuras colaboraciones.

- Iniciar un espacio de reflexión sobre la Gestión y el Desarrollo Turístico Sostenible como una actividad que genera beneficio sin comprometer el presente y el futuro del patrimonio natural y cultural de nuestras regiones y países que conforman esta red.

- Promover la presentación, discusión y enriquecimiento de propuestas de investigación que sigan la línea de Gestión y Desarrollo Turístico Sostenible.

- Compartir resultados de investigación y favorecer el intercambio de experiencias con respecto a la línea de Gestión y Desarrollo Turístico Sostenible.

- Generar artículos para la edición de revistas científicas de las universidades y memorias que agrupen las diferentes ponencias y reflexiones de los académicos y demás actores participantes en los congresos desarrollados por la red.

- Destacar las posibilidades del desarrollo sostenible en el área de turismo con el fin de aliviar la pobreza y permitir que los grupos más vulnerables logren mejorar sus condiciones de vida a través de las fuentes que generen ingresos, tomando en cuenta sus destrezas y habilidades en conjunto con el potencial histórico-cultural -social y ambiental de las comunidades que logren participar en este proyecto.

- Elaborar planes conjuntos entre las universidades integrantes de la red y éstas con los encargados de diseñar políticas de turismo a saber los Institutos de Turismo, así como con los Ministerios de Comercio y de Relaciones Exteriores, con el fin de obtener apoyo institucional que permita crear las condiciones necesarias para que las actividades de turismo sostenible se desarrollen con éxito.

- Establecer programas de investigación y de capacitación en cada una de las universidades integrantes de la red para que el turismo sea visto como propuesta de desarrollo comunitario que permita generar una mayor cantidad de empleos. 
- Diseñar planes estratégicos de coordinación institucional con la participación de cada universidad integrante de la red para ayudar a resolver las necesidades prioritarias de las comunidades.

\section{Plan de docencia}

Las universidades integrantes de las red Galeón, titulada Gestión y Desarrollo Turístico Sostenible elaboraron de forma conjunta un módulo docente de 60 horas lectivas por cada año de duración de la red. El primer módulo se desarrolló en La Corporación Universitaria del Meta (UNIMETA) (Colombia) en el año 2008 y el otro en la Universidad de Panamá (Panamá) en el año 2009.

\section{Contenidos (Bloques temáticos)}

La primera actividad que desarrolló la red en agosto del 2008 se realizó en la Corporación Universitaria del Meta (UNIMETA) (Colombia), universidad coordinadora del congreso titulado "Congreso Iberoamericano sobre Gestión y Desarrollo Turístico Sostenible”, el cual se realizó en torno a los siguientes ejes temáticos: Clusters Turísticos; Marketing Turístico; Gestión Turística; Planificación y Desarrollo Turístico Sostenible; Turismo y Medio Ambiente, e Identidad, Cultura y Territorio.

La segunda actividad que desarrolló la red Galeón en la Universidad de Panamá en el año 2009 fue un Simposio de 60 horas distribuidas de la siguiente manera: durante tres días en auditorios de la universidad y dos días para desarrollar un laboratorio de campo en un punto estratégico cercano a la ciudad de Panamá. Los bloques temáticos a desarrollar fueron los de Urbanismo y Desarrollo Turístico; Globalización y Desarrollo Turístico; los Municipios Turísticos: Gestión y Administración; Salud y Promoción Turística, y la visita a un sitio estratégico para identificar e inventariar áreas de potencial turístico (Canal de Panamá).

\section{Memoria justificativa}

El tema del desarrollo del turismo ha atraído la atención de investigadores y de profesionales del ramo; al mismo tiempo, ha producido una buena cantidad de encuentros académicos a nivel mundial. Sin embargo, y a pesar de los esfuerzos, el debate académico no ha sido suficiente sobre todo cuando se observa en Latinoamérica, principalmente, un mínimo interés por la realización de estudios donde se examine la gestión y sostenibilidad de los destinos turísticos. Esto cobra también relevancia cuando se advierte la existencia de numerosos problemas como ambientes dañados, culturas destrozadas y graves conflictos sociales en los propios lugares receptores de turismo.

Debido a tales problemas se cuestiona el éxito del desarrollo turístico implementado desde el enfoque económico. Se argumenta una propuesta más integral que contemple la perspectiva ambiental y los aspectos socioculturales y hasta políticos. Los análisis de los investigadores apuntan a que el problema del equilibrio entre uso y preservación de los recursos naturales y culturales, y mejoramiento del nivel de vida de las comunidades locales no puede ser visto de forma aislada o parcializada, tal como sucede cuando se quiere explicar la plusvalía de las poblaciones residentes en función de una simple medición económica.

En consecuencia con lo anterior, la Red Temática de Docencia en Turismo Galeón, titulada Gestión y Desarrollo Turístico Sostenible, configuró en torno a los bloques temáticos propuestos en el Plan Docente, para el primer año (2008) un congreso y para el segundo año (2009) un simposio de debate de ideas entre expertos reconocidos del ramo que contribuyeron al desarrollo sostenible, además de propiciar espacios de enseñanza con profesores prestigiosos y reconocidos (en nuestro caso, peruanos, panameños y colombianos), que permitieron actualizar y complementar los conocimientos de los profesores y estudiantes participantes, como elevar la calidad académica de los programas docentes de las universidades que colaboran en la citada red.

Consideramos pertinentes estos objetivos dado que el movimiento turístico muestra cada vez más un incremento económico en nuestras balanzas de pago.

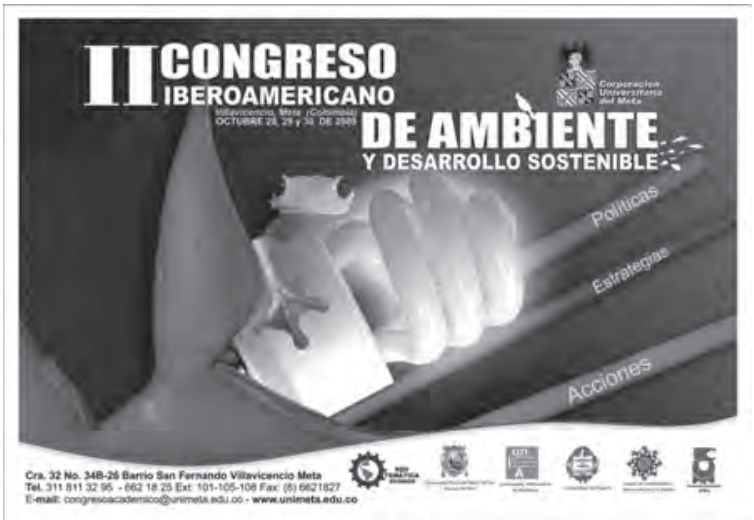


El volumen de turistas que visitan cada uno de los países (Panamá, Perú y Colombia) evidencia un crecimiento constante por lo que se hace necesario crear equipos de investigación y ofrecer una mejor formación y capacitación a un personal calificado académica y técnicamente.

Es necesario ver al turismo como un eje que articule las otras actividades que se dan en las comunidades y que a la vez actúe como promotor, capaz de beneficiar a las mismas. A través de políticas de desarrollo, propias de la comunidad, se puede crear una cultura turística a largo plazo, impulsadas por una planificación estratégica.

De manera directa los involucrados en cada una de las actividades desarrolladas por la red fueron los estudiantes de la Escuela de Ciencias Administrativas, claro que ellos no fueron los únicos interesados, ya que son temáticas que no solo involucran a estos participantes de la disciplina administrativa, sino que estuvo abierto a estudiantes de las Facultades de Ambiental, Arquitectura, entre otras.

Cada uno de los participantes de los módulos contaron con otra opción formativa, donde se les proporcionaron conocimientos y herramientas para la real comprensión y explicación de la compleja y contradictoria realidad social y turística. Previo a la realización del Congreso y Simposio, los estudiantes de las universidades de la red contaron con las lecturas necesarias (bibliohemerografía) que les permitieron estimular un pensamiento crítico y complejo.

\section{RED ECOBIOS}

La Red Temática Ecobíos, titulada Desarrollo Sostenible: Estrategias, Políticas y Acciones, se conforma de acuerdo a las bases establecidas en la Resolución de 11 de julio del 2007 del Presidente del Grupo de Universidades Iberoamericanas La Rábida por la que se convocan ayudas para redes temáticas de docencia, a las cuales debe responder en sus características, objetivos, configuración $\mathrm{y}$ fines.

\section{Composición (universidades)}

- Corporación Universitaria del Meta (UNIMETA) de Colombia (Coordinadora).

- Universidad Nacional Mayor de San Marcos (Perú).

- Universidad de Panamá (Panamá).

\section{Objetivo general.}

La Red Temática ECOBíOS, titulada "El Desarrollo Sostenible: Estrategias, Políticas y Acciones", tiene como objetivo principal analizar y afianzar los criterios según los cuales el desarrollo y la conservación deben ser indisolublemente ligados para la satisfacción de las necesidades socioeconómicas actuales y futuras.

\section{Objetivos específicos}

- Analizar experiencias significativas derivadas de la planificación y gestión inherentes a los recursos naturales y a los sectores productivos tradicionales, interpretando sus interrelaciones, alcances y posibles factores correctivos.

- Identificar las prioridades de acción y mecanismos para la utilización de investigaciones básicas aplicadas, así como tecnologías apropiadas que contemplen la verdadera oferta ambiental, potencialidades y limitaciones de uso frente a aspectos socio-económicos y culturales, fundamentalmente para la planificación y gestión del desarrollo.

- Plantear los lineamientos y la estructura institucional conducente a la elaboración de una estrategia de desarrollo sostenible y conservación.

- Conocer los componentes del desarrollo sostenible y potenciar su utilización a nivel de sistemas productivos.

- Desarrollar acciones de fortalecimiento institucional, educación y formación social, planificación regional y binacional, información, seguimiento y evaluación del desarrollo sostenible que aseguren su adecuada implementación y consolidación.

- Desarrollar, promover e implementar iniciativas de uso sostenible de la biodiversidad.

- Evaluar el ordenamiento territorial como una estrategia de desarrollo sostenible.

- Evaluar las acciones a nivel de política estatal y su impacto en las condiciones medioambientales y la interacción sociedad-naturaleza.

- Evaluar el papel de la educación ambiental en la internalización y construcción social de los elementos ambientales en la vida cotidiana.

- Evaluar y ponderar el uso de los instrumentos de gestión ambiental utilizados como regula- 
dores de las acciones sociales de los grupos, frente a la racionalidad económica.

\section{Plan de docencia}

Las universidades integrantes de la red Ecobíos, titulada "Desarrollo Sostenible: Estrategias, Políticas y Acciones", elaboraron de forma conjunta un módulo docente de 60 horas lectivas que se desarrollaron en dos universidades iberoamericanas de la red, durante un periodo de dos años.

\section{Contenidos (Bloques temáticos)}

En el año 2008 se desarrolló un seminario en la Universidad de Panamá durante seis días que contempló cinco temas centrales, de importancia para el desarrollo sostenible y la gestión ambiental, incluyendo una gira por zonas intervenidas, donde confluyeron distintos actores en el escenario natural con los siguientes temas: Políticas Ambientales y Desarrollo Humano Sostenible; Ambiente y Canal de Panamá; Discurso Político y Medio Ambiente; Construcción Social del Ambiente para el Desarrollo Sostenible: explicaciones teóricas y metologías para el estudio y evaluación de la relación sociedad-naturaleza.

En el año 2009 se desarrolló el Módulo Docente en la Corporación Universitaria del Meta (UNIMETA) en Colombia en la ciudad de Villavicencio, el cual constó de seis bloques temáticos que se desarrollaron durante tres días del mes de agosto en dos auditorios simultáneamente; cada temática estuvo conformada de ponencias pertinentes a los temas a tratar como fueron los de Desarrollo Sostenible, Conservación y Economía; Ciencia y Tecnología como Componentes del Desarrollo Sostenible y la Conservación; Estrategias para la Conservación y el Desarrollo; Políticas, Educación Ambiental, Ciencia y Tecnología; Biodiversidad, Desarrollo y Conservación, y los Esquemas de Ordenamiento Territorial y el Desarrollo Sostenible.

\section{Memoria justificativa}

Es prioritario aunar esfuerzos para que se establezcan y refuercen los programas tendientes a la preservación y conservación de los ecosistemas altamente frágiles y/o de elevada productividad, o que no han recibido la debida atención en cuanto a su manejo, en especial los páramos andinos, los manglares y ecosistemas costeros, praderas submarinas y arrecifes coralinos y, en general, los humedales. Merecen particular mención en el caso colombiano, los ecosistemas forestales de la región biogeográfica del Chocó.

Es necesario que tanto organizaciones nacionales como internacionales promuevan el desarrollo de nuevas metodologías y enfoques que vinculen de manera dinámica la economía con la ecología a través de una mejor comprensión de la acertada evaluación de la oferta y la demanda natural de bienes y servicios, con sus limitaciones y potencialidades.

Se requiere adoptar y mantener una férrea voluntad política para evitar la destrucción de los recursos naturales renovables, donde los beneficios para la humanidad son escasos y los costos para los ecosistemas son demasiado altos; tal es el ejemplo de muchas zonas de Colombia y de otros países. Debemos aspirar a no reproducir los errores que han sido patentes en las sociedades industriales, objetivo que requiere de una activa intervención del Estado en el aprovechamiento racional de los recursos naturales renovables y no renovables, de manera que se garantice el desarrollo de un potencial productivo sin sacrificar la conservación y el equilibrio de nuestro patrimonio natural.

El concepto de desarrollo sostenible ha sido definido por la Comisión Mundial para el Medio Ambiente y el Desarrollo, integrada por las Naciones Unidas para su estudio y análisis, como "la satisfacción de las necesidades del presente sin comprometer la capacidad de las futuras generaciones para satisfacer sus propias necesidades". El concepto se insinúa también en las palabras de la doctora Gro Harlem Brundtland, Primera Ministra de Noruega, al aceptar del Secretario General de las Naciones Unidas el encargo de presidir esta Comisión Mundial: "acepto el reto de afrontar el futuro", dijo, "y de salvaguardar los intereses de las generaciones venideras".

\section{¿CÓMO LLEGAR AL DESARROLLO SOSTENIBLE?}

Nadie lo sabe a ciencia cierta; se conocen los objetivos pero se ignora con detalle los procedimientos, las acciones y las estrategias para alcanzarlos. Según la conocida obra "Nuestro Futuro Común”, publicada como resultado por la ya citada comisión y sus asesores, el logro del desarrollo sostenible requiere de las siguientes condiciones: 
- Sistemas políticos que aseguren la efectiva participación ciudadana en la toma de decisiones.

- Sistemas económicos que sean capaces de generar excedentes y conocimientos técnicos de manera permanente y confiable.

- Sistemas sociales que presenten soluciones a las tensiones resultantes del desarrollo no armónico.

- Sistemas de producción que respeten la obligación de preservar la base ecológica para el desarrollo.

- Sistemas tecnológicos que puedan buscar continuamente nuevas soluciones.

- Sistemas internacionales que fomenten patrones sostenibles de comercio y finanzas.

- Sistemas administrativos que sean flexibles y tengan la capacidad de autocorrección.

Los anteriores requisitos están siendo objeto de profundo debate por su naturaleza, sus implicaciones y el esfuerzo que demandan de los gobiernos, a nivel individual y colectivo, para ajustarse a ellos. Sin embargo, este es un esfuerzo que vale la pena hacer, ya que las alternativas del desarrollo sostenible tienen un costo ecológico, económico y social mucho mayor.

\section{CONCLUSIONES}

- Las redes temáticas de docencia tienen como objetivo principal el intercambio de conocimientos entre grupos de investigación y la potenciación de la cooperación como método de trabajo.
- La mayoría de redes temáticas subvencionan los gastos de transporte y movilidad, cuyo porcentaje es hasta el $75 \%$ del gasto total de las conferencias internacionales, dejando un $25 \%$ de viáticos y manutención a la universidad organizadora del evento.

- A la fecha, hemos presentado un Proyecto de Renovación para la Red GALEON (Turismo) ya que las redes pueden renovarse por un año.

- Algo muy importante de los resultados es que generan publicaciones que no solamente benefician a una sola facultad o universidad sino a varias universidades de los países confortantes de la red y que van a las bibliotecas para beneficio de docentes, investigadores y alumnos.

- La universidad no debería solamente pertenecer a una, dos o tres redes de diversos temas, sino a más redes que también sean afines a cada una de las Escuela Académico Profesionales de Administración, Administración de Turismo y Administración de Negocios Internacionales.

- Es muy importante tener en cuenta los programas y las líneas de investigación del Instituto de Investigaciones de la Facultad de Ciencias Administrativas para buscar ayudas en redes temáticas afines a estas líneas.

- Podemos pertenecer a otras redes temáticas para continuar ampliando nuestros horizontes internacionales y lograr que nuestra facultad se inserte en el mundo de la investigación con otros países que en un inicio serán latinoamericanos, pero en un futuro será ya con Estados Unidos y por qué no con Europa, traspasando la barrera del idioma. 